DRAFT VERSION JuLY 17, 2018

Preprint typeset using $\mathrm{LAT}_{\mathrm{E}} \mathrm{X}$ style emulateapj v. 12/16/11

\title{
NO STRONG GEOMETRIC BEAMING IN THE ULTRALUMINOUS NEUTRON STAR BINARY NGC 300 ULX-1 (SN 2010DA) FROM SWIFT AND GEMINI
}

\author{
B. Binder ${ }^{1}$, E. M. Levesque ${ }^{2}$, T. Dorn-Wallenstein ${ }^{2}$ \\ Draft version July 17, 2018
}

\begin{abstract}
We have obtained near-simultaneous Swift/XRT imaging and Gemini GMOS spectroscopy for the ultraluminous X-ray source (ULX) NGC 300 ULX-1 (formerly designated SN 2010da). The observed $\mathrm{X}$-ray emission is consistent with an inhomogeneous wind that partially obscures a central, bright inner accretion disk. We simultaneously fit eleven $0.3-10 \mathrm{keV}$ spectra obtained over a $\sim 1$ year time period (2016 April to 2017 July) using the same partial covering model, and find that although the covering fraction varies significantly (from $78 \%$ to consistent with $0 \%$ ), the unabsorbed luminosity remains essentially constant across all observations $\left(2-6 \times 10^{39} \mathrm{erg} \mathrm{s}^{-1}\right)$. A relatively high $0.3-10 \mathrm{keV}$ fractional variability amplitude $\left(F_{\text {var }}\right)$ of $\sim 30 \%$ is observed in all eleven observations. Optical spectra from Gemini exhibit numerous emission lines (e.g., $\mathrm{H} \alpha, \mathrm{H} \beta$, He II $\lambda 4686$ ) which suggest that the neutron star primary is photoionizing material in the immediate vicinity of the binary. We compare the He II $\lambda 4686$ line luminosity $\left(\sim 7-9 \times 10^{35} \mathrm{erg} \mathrm{s}^{-1}\right)$ to the contemporaneous soft X-ray emission and find the X-ray emission is broadly consistent with the observed He II line luminosity. The combination of our X-ray observations and optical spectroscopy suggest that geometric beaming effects in the ULX1 system are minimal, making ULX-1 one of only a few bona fide ULXs to be powered by accretion onto a neutron star.

Subject headings: X-rays: binaries, individual (SN 2010da, NGC 300 ULX-1) - accretion, accretion disks - ultraluminous X-ray sources
\end{abstract}

\section{INTRODUCTION}

It is now well-established that the optical transient SN 2010da (Monard 2010; Immler et al. 2010) in NGC 300 was a supernova "impostor" (Chornock \& Berger 2010; Elias-Rosa et al. 2010a, b), and likely a young (<5 Myr; Binder et al. 2011, 2016) high-mass X-ray binary (HMXB). Several multiwavelength studies (from X-ray to infrared) have been conducted to examine the nature of the binary components (Binder et al. 2016; Villar et al. 2016; Lau et al. 2016), and recently deep XMM-Newton + NuSTAR observations have confirmed the neutron star origin of the X-ray emission (Carpano et al. 2018; Walton et al. 2018; Kosec et al. 2018). The most likely scenario is that SN 2010da hosts a neutron star primary with a spin period $P_{S} \sim 31.6$ s and $\dot{P}=-5.4 \times 10^{-7} \mathrm{~s}^{-1}$ (Carpano et al. 2018) and a supergiant companion (Lau et al. 2016), possibly a yellow- or red-supergiant (RSG) entering a blue-loop phase of its evolution (Villar et al. 2016). Optical spectroscopy (Chornock \& Berger 2010; Elias-Rosa et al. 2010b; Chornock et al. 2011; Villar et al. 2016) has revealed complex environment. Much of the dust that enshrouded the progenitor system (Khan et al. 2010) was destroyed in the initial optical/X-ray outburst (Brown 2010; Prieto et al. 2010), and recent observations suggest that new dust is actively forming from the supergiant donor star (Lau et al. 2016). The high X-ray luminosities $\left(\sim 10^{39} \mathrm{erg} \mathrm{s}^{-1}\right)$ recently observed in the XMM and $N u S T A R$ have

\footnotetext{
${ }^{1}$ Department of Physics \& Astronomy, California State Polytechnic University, $3801 \mathrm{~W}$. Temple Avenue, Pomona, CA 91768

${ }^{2}$ University of Washington, Department of Astronomy, Box 351580, Seattle, WA 98195
}

earned SN 2010da a new designation: NGC 300 ULX-1.

Ultraluminous X-ray sources (ULXs) are off-nuclear Xray sources with luminosities exceeding the Eddington limit of a $10 M_{\odot}$ black hole in the $0.3-10 \mathrm{keV}$ energy band (for a review, see Feng \& Soria 2011). The extreme Xray luminosities are expected to arise from 'supercritical' accretion (King 2009; Bachetti et al. 2014), where large scale-height, optically thick winds are radiatively driven to large radii, revealing a hot accretion disk (King 2004; Poutanen et al. 2007). Inclination angle, therefore, plays a major role in the appearance of ULXs (Middleton et al. 2011, 2014; Sutton et al. 2013), and beaming effects may amplify the X-ray emission (particularly in the hard band King 2009; Poutanen et al. 2007). Clumps and inhomogeneities in the winds can imprint variability in the $\mathrm{X}$ ray emission, which is likewise dependent on inclination angle (Takeuchi et al.|2013, 2014; Middleton et al.|2011; Heil et al. 2009; Sutton et al. 2013).

We present the results of recent monitoring campaign of NGC 300 ULX-1/SN 2010da (hereafter, ULX-1) by the Neil Gehrels Swift Observatory (hereafter, Swift) combined with ground-based optical spectroscopy with the Gemini Observatory. Throughout this work, we assume a distance to NGC 300 of 2.0 Mpc (Dalcanton et al. 2009, corresponding to a redshift $z=0.00047$ ), and a column of neutral absorption fixed at the Galactic column $\left(N_{\mathrm{H}}\right.$ $=4.09 \times 10^{20} \mathrm{~cm}^{-2}$; Kalberla et al. 2005).

\section{OBSERVATIONS}

\subsection{Swift/XRT}

Swift/XRT (Burrows et al. 2004) observations were obtained from 2016 April 14 through 2017 April 22 through the Swift archive (Evans et al. 2009), and our team acquired three new target-of-opportunity observations on 
TABLE 1

Summary of Gemini ObServations

\begin{tabular}{ccccc}
\hline \hline $\begin{array}{c}\text { Date } \\
(\mathrm{UT})\end{array}$ & $\begin{array}{c}\text { Grating } \\
(1)\end{array}$ & $\begin{array}{c}\lambda_{C} \\
(\AA)\end{array}$ & $\begin{array}{c}\text { Exp. Time } \\
(\mathrm{s})\end{array}$ & $\begin{array}{c}\text { Resolution } \\
(\AA)\end{array}$ \\
\hline 2017 Jun 19 & B600 & 5000 & $2 \times 600$ & 3 \\
& B600 & 5050 & $2 \times 600$ & 3 \\
& R400 & 7000 & $2 \times 600$ & 4 \\
2017 Jul 02 & B600 & 5000 & $2 \times 600$ & 3 \\
& B600 & 5050 & $2 \times 600$ & 3 \\
& R400 & 7000 & $2 \times 600$ & 4 \\
& R400 & 7050 & $2 \times 600$ & 4 \\
2017 Jul 18 & R400 & 7000 & $2 \times 600$ & 4 \\
& R400 & 7050 & $2 \times 600$ & 4 \\
2017 Jul 30 & B600 & 5000 & $2 \times 600$ & 3 \\
& B600 & 5050 & $2 \times 600$ & 3 \\
\hline \hline
\end{tabular}

2017 July 2, 6, and 12 to coincide with our Gemini observations. All observations were performed in Photon Counting mode (Hill et al. 2004). The data were produced using standard procedures (XRTPIPELINE) using the FTOOLS in the HEASOFT package ( $v$ 6.17).

The location of the X-ray source corresponding to ULX-1 has been measured with high precision by Chan$d r a$, with a right ascension of 00:55:04.9 and a declination of $-37: 41: 44.0$. Spectra were extracted in each observations where an X-ray source was detected near these coordinates with a signal-to-noise $\geq 3$; we used a circular aperture with a $40^{\prime \prime}$ radius centered on the Chandra position of ULX-1. The background was extracted from a circular aperture (radius of $80^{\prime \prime}$ ) offset from the ULX1 position in a region with no obvious contaminating X-ray sources. Figure 1 shows an example Swift/XRT image, with our extraction regions superimposed. Ancillary response files were generated for each spectrum with XRTMKARF using the spectral redistribution matrix v0153.

\subsection{Gemini Spectroscopy}

We obtained time-resolved optical spectra of ULX-1 using the Gemini Multi-Object Spectrograph on Gemini South (Hook et al. 2004; Gimeno et al. 2016). The observations were carried out in queue mode during June and July of 2017 and were originally designed to be executed in three separate visits, each with complete wavelength coverage, spaced 5 and 10 days apart respectively. Unfortunately, poor weather at Gemini prevented this execution, and we instead obtained four different spectra with full or partial wavelength coverage spanning a 42day period. Our final set of observations is summarized in Table 1 .

Our spectra were taken with the $0.5^{\prime \prime} \times 330^{\prime \prime}$ slit, using the B600 grating centered at $5000 \AA$ and the R400 grating (with the GG455 blocking filter) centered at $7000 \AA$ for a total wavelength coverage of $\sim 3600 \AA$ to $9000 \AA$; the grating centers shifted by $+50 \AA$ for half of our observations to avoid the detector gaps. Image quality during these observations was $\geq 85 \%$ 2 , and the data were taken at a mean airmass of 1.1. Standard quartz lamp and

${ }^{3}$ See http://heasarc.gsfc.nasa.gov/docs/heasarc/caldb/swift 2 Our image quality criteria correspond to a

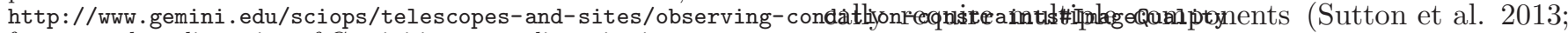
for a complete discussion of Gemini image quality criteria (Middleton et al. [2015). (Carpano et al. (2018) found

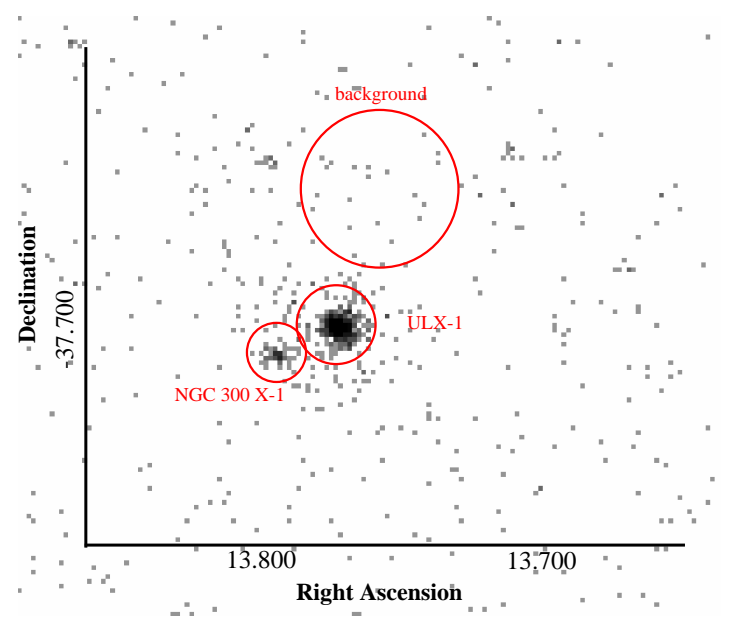

FIG. 1.- The 0.3-10 keV Swift/XRT image of ULX-1 (SN 2010da) from ObsID 00049834010. The source and background extraction regions are shown, as well as the location of the nearby X-ray source NGC 300 X-1.

CuAr arc lamp observations were taken for flat-field and wavelength calibrations.

The data were reduced using the gemini package in IRAF Gemini Observatory \& AURA 2016). Data read out through the 12 amplifiers on the three GMOS-S chips were mosaiced together with the gprepare task. A bias image and flat field images for each grating were created from the gbias and gsflat tasks, and the data were reduced with gsreduce. Two-dimensional wavelength solutions in each grating were created with gswavelength and applied with gstransform. We used gsskysub on the two dimensional images to account for telluric features in the data. One-dimensional spectra were traced, extracted, and background-subtracted with gsextract. We also observed the spectrophotometric standard star Hiltner 600 (aka H600/HD 289002, Baldwin \& Stone 1984) with the same instrument setup on 2017 March 3rd - unfortunately we were unable to obtain observations of $\mathrm{H} 600$ on the same night as our observations of ULX-1 due to poor weather. We derive the sensitivity function for each grating using gsstandard and applied it to the data with gscalibrate. Finally, the data from individual nights were combined using gemscombine.

The resulting flux-calibrated GMOS spectra are shown in Figure 2 and line identifications and fluxes (corrected for a foreground reddening of $\mathrm{E}(\mathrm{B}-\mathrm{V})=0.011$; Schlafly \& Finkbeiner 2011) are listed in Table 2. The wavelengths in both Figure 2 and Table 2 are in the observer frame (as was done in Villar et al. 2016). The redshift to NGC 300 is very small $\left(\sim 141 \mathrm{~km} \mathrm{~s}^{-1}\right)$, and it is thus impossible to clarify whether the observed shifts in the lines relative to the rest wavelength can be attributed to the velocity of NGC 300 itself, the motion of ULX-1 within NGC 300, or the velocity of the emission source within the system.

\section{X-RAY ANALYSIS \\ 3.1. Spectral Modeling}

X-ray emission from ULX-1 was detected with a signal-to-noise $\geq 3$ in all 11 Swift/XRT observations obtained since 2016. High-quality ULX spectra typi- 


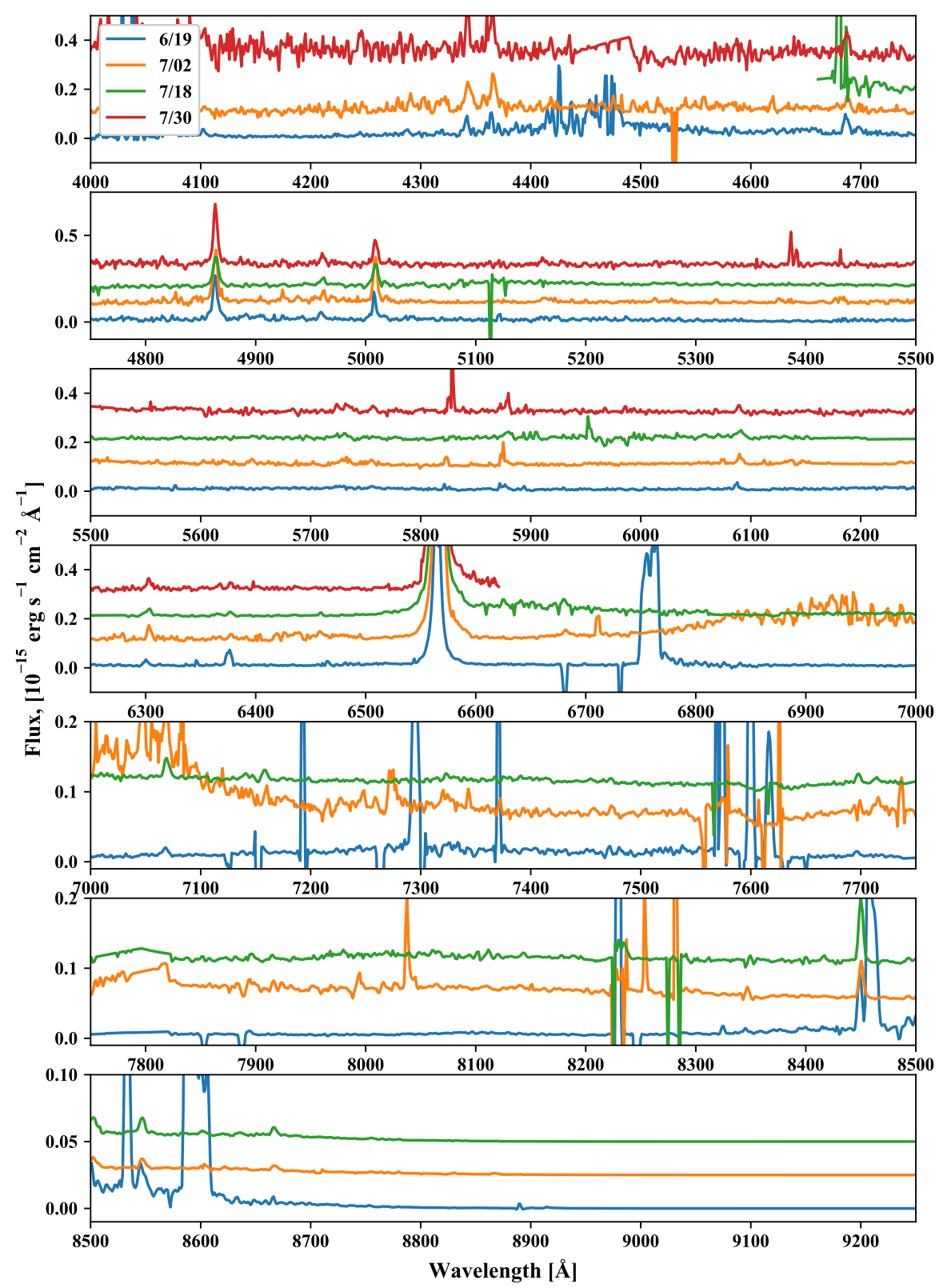

FIG. 2.- The four Gemini GMOS spectra of ULX-1 (SN 2010da). Flux levels have an arbitrary offset for clarity.

that variations in the observed flux from ULX-1 in $X M M-N e w t o n$ and $N U S T A R$ could be interpreted in changes in absorbing column only. We therefore simultaneously fit all eleven $0.3-10 \mathrm{keV}$ Swift spectra using XSPEC (Arnaud 1996) v.12.9.1 with a single model (tbabs*pcfabs*(diskbb+nthcomp)); only the covering fraction was allowed to vary between each observation. The neutral absorbing column lower limit was set to the Galactic line-of-sight value $\left(4.09 \times 10^{20} \mathrm{~cm}^{-2}\right.$; Kalberla et al. 2005). We used a multicolor disk blackbody component to model the optically thick wind emission, and nthcomp has been used previously to describe the harder X-ray emission originating in the inner disk (e.g., Middleton et al. 2015, while other authors have used a simple power law to represent this component). Due to the low number of counts, we fit the unbinned spectra using $C$-statistics, and the Pearson $\chi^{2}$ statistic was used as a "test" statistic for comparison purposes only.

The best-fit spectral model parameters are given in Table 3. The fit parameters were generally consistent with those found by Carpano et al. (2018), although the Swift data require substantial intrinsic absorption associated with the partial covering model component $\left(>32.2 \times 10^{22}\right.$ $\mathrm{cm}^{-2}$ ). A similarly high absorbing column was required to adequately fit the 2010 XMM observations that were obtained shortly after the initial outburst. This result is consistent with the recent multiwavelength SED modeling by Lau et al. (2016) and Villar et al. (2016), which suggested that dust was actively re-forming in the vicinity of ULX-1. Details about the eleven individual observations used in the spectral fitting, with the observationspecific best-fit partial covering fraction and unabsorbed luminosities, are presented in Table 4 . 
TABLE 2

Optical Line IDENTifications

\begin{tabular}{|c|c|c|c|c|c|c|c|c|c|c|c|c|}
\hline Date & Line ID & Shape $^{a}$ & $\begin{array}{l}\lambda_{\mathrm{c}}^{b} \\
(\AA) \\
(4)\end{array}$ & $\begin{array}{c}\text { Flux }\left(10^{-15} \text { erg }\right. \\
\left.\mathrm{s}^{-1} \mathrm{~cm}^{-2} \AA^{-1}\right) \\
(5)\end{array}$ & $\begin{array}{c}\text { FWHM } \\
\left(\mathrm{km} \mathrm{s}^{-1}\right) \\
(6)\end{array}$ & $\begin{array}{c}\text { Eq. Width } \\
(\AA) \\
(7)\end{array}$ & $\begin{array}{c}\text { Line ID } \\
(8)\end{array}$ & $\begin{array}{c}\text { Shape }^{a} \\
(9)\end{array}$ & $\begin{array}{l}\lambda_{\mathrm{c}}^{b} \\
(\AA) \\
(10)\end{array}$ & $\begin{array}{c}\text { Flux }\left(10^{-15} \text { erg }\right. \\
\left.\mathrm{s}^{-1} \mathrm{~cm}^{-2} \AA^{-1}\right) \\
(11)\end{array}$ & $\begin{array}{c}\text { FWHM } \\
\left(\mathrm{km} \mathrm{s}^{-1}\right) \\
(12)\end{array}$ & $\begin{array}{c}\text { Eq. Width } \\
(\AA) \\
(13)\end{array}$ \\
\hline June 19 & & $\mathrm{G}$ & 4102.44 & 0.099 & 231 & -7.346 & \multirow{3}{*}{$\mathrm{H} \gamma$} & $G$ & 4341.65 & 0.338 & 305 & -25.79 \\
\hline July 2 & $\mathrm{H} \delta$ & G & 4103.80 & 0.134 & 323 & -8.034 & & G & 4343.46 & 0.863 & 515 & -44.54 \\
\hline July 30 & & & $\ldots$ & $\ldots$ & $\ldots$ & $\ldots$ & & G & 4342.52 & 0.756 & 211 & -13.22 \\
\hline June 19 & {$[\mathrm{O}$ III $]+$} & G & 4364.25 & 0.243 & 185 & -8.522 & \multirow{3}{*}{ He II } & $\mathrm{G}$ & 4686.71 & 0.430 & 338 & -21.2 \\
\hline July 2 & {$[\mathrm{Fe}$ IX $]+$} & G & 4366.16 & 0.688 & 354 & -21.89 & & $\mathrm{G}$ & 4688.15 & 0.318 & 342 & -24.3 \\
\hline July 30 & Fe II & G & 4364.34 & 0.640 & 149 & -6.623 & & G & 4687.07 & 0.587 & 355 & -15.4 \\
\hline June 19 & \multirow{4}{*}{$\mathrm{H} \beta$} & $\mathrm{L}$ & 4863.16 & 1.967 & 280 & -205.2 & \multirow{4}{*}{$\mathrm{O}$ III] } & $\mathrm{G}$ & 4959.57 & 0.238 & 296 & -16.86 \\
\hline July 2 & & $\mathrm{~L}$ & 4863.45 & 2.103 & 253 & -109.5 & & G & 4961.73 & 0.207 & 185 & -9.865 \\
\hline July 18 & & $\mathrm{~L}$ & 4863.84 & 1.479 & 293 & -306.5 & & $\mathrm{G}$ & 4960.89 & 0.207 & 324 & -16.14 \\
\hline July 30 & & $\mathrm{~L}$ & 4863.38 & 2.333 & 240 & -76.2 & & G & 4960.88 & 0.334 & 268 & -6.50 \\
\hline June 19 & \multirow{4}{*}{ [O III] } & $\mathrm{L}$ & 5007.77 & 0.875 & 186 & -73.3 & \multirow{4}{*}{ 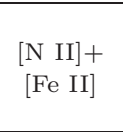 } & $G$ & 5755.06 & 0.038 & 251 & -3.583 \\
\hline July 2 & & $\mathrm{G}$ & 5008.8 & 1.196 & 253 & -54.24 & & G & 5755.36 & 0.104 & 243 & -11.98 \\
\hline July 18 & & $\mathrm{G}$ & 5008.86 & 0.837 & 350 & -461.8 & & & $\ldots$ & $\ldots$ & $\ldots$ & $\ldots$ \\
\hline July 30 & & $\mathrm{G}$ & 5008.93 & 0.682 & 249 & -12.94 & & $\mathrm{G}$ & 5756.86 & 0.018 & 305 & -8.587 \\
\hline June 19 & \multirow{4}{*}{ He I } & $\mathrm{c}$ & $\ldots$ & $\ldots$ & $\ldots$ & $\ldots$ & \multirow{4}{*}{$\mathrm{Fe}$ VIII] } & $\mathrm{G}$ & 6087.1 & 0.094 & 171 & -11.18 \\
\hline July 2 & & $\mathrm{c}$ & $\ldots$ & $\ldots$ & $\ldots$ & $\ldots$ & & G & 6090.11 & 0.174 & 245 & -13.54 \\
\hline July 18 & & G & 5879.01 & 0.293 & 483 & -30.22 & & G & 6090.45 & 0.301 & 423 & -14.00 \\
\hline July 30 & & $\mathrm{c}$ & $\ldots$ & $\ldots$ & $\ldots$ & $\ldots$ & & $\mathrm{G}$ & 6089.17 & 0.150 & 225 & -8.665 \\
\hline June 19 & \multirow{4}{*}[\begin{array}{ll}{\mathrm{O}}&{\mathrm{I}}\end{array}]{} & $G$ & 6300.33 & 0.147 & 285 & -26.58 & \multirow{4}{*}[\mathrm{Fe}\mathrm{X}]{} & $G$ & 6375.71 & 0.360 & 246 & -34.98 \\
\hline July 2 & & G & 6303.35 & 0.271 & 202 & -19.19 & & G & 6377.66 & 0.115 & 255 & -8.165 \\
\hline July 18 & & G & 6302.66 & 0.151 & 278 & -10.6 & & G & 6377.09 & 0.085 & 211 & -6.445 \\
\hline July 30 & & G & 6302.91 & 0.181 & 194 & -6.267 & & G & 6377.24 & 0.114 & 179 & -4.157 \\
\hline June 19 & \multirow{4}{*}{$\mathrm{H} \alpha$} & $\mathrm{L}$ & 6564.57 & 7.846 & 343 & -453.7 & \multirow{4}{*}{$\mathrm{He} \mathrm{I}$} & $\mathrm{G}$ & 7067.89 & 0.080 & 304 & -11.14 \\
\hline July 2 & & $\mathrm{~L}$ & 6566.56 & 19.52 & 299 & -961.7 & & G & 7068.98 & 0.377 & 219 & -4.52 \\
\hline July 18 & & $\mathrm{~L}$ & 6566.17 & 26.43 & 298 & -696.2 & & G & 7069.2 & 0.167 & 235 & -8.247 \\
\hline July 30 & & $\mathrm{~L}$ & 6565.8 & 31.38 & 257 & -905.7 & & & $\ldots$ & $\ldots$ & $\ldots$ & $\ldots$ \\
\hline June 19 & \multirow{2}{*}[\mathrm{Ca}\mathrm{II}]{} & & $\ldots$ & $\ldots$ & $\ldots$ & 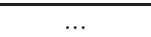 & \multirow{2}{*}[\mathrm{Fe}\mathrm{XI}]{} & $\mathrm{G}$ & 7894.32 & 0.033 & 180 & -5.371 \\
\hline July 18 & & G & 7323.15 & 0.033 & 140 & -1.961 & & G & 7896.61 & 0.082 & 172 & -4.518 \\
\hline June 19 & \multirow{3}{*}{ O I } & $G$ & 8448.95 & 0.341 & 151 & -22.66 & \multirow{3}{*}{$\begin{array}{c}\mathrm{Ca} \mathrm{II}+ \\
\mathrm{Pa} 8500\end{array}$} & $G$ & 8500.65 & 0.051 & 114 & -3.931 \\
\hline July 2 & & G & 8450.46 & 0.247 & 157 & -34.12 & & G & 8502.96 & 0.045 & 242 & -9.288 \\
\hline July 18 & & G & 8450.11 & 0.452 & 157 & -39.83 & & G & 8503.0 & 0.067 & 252 & -8.854 \\
\hline June 19 & $\mathrm{Ca} \mathrm{II+}$ & $\mathrm{G}$ & 8546.89 & 0.154 & 241 & -8.499 & \multirow{3}{*}{$\begin{array}{c}\mathrm{Ca} \mathrm{II}+ \\
\mathrm{Pa} 8664\end{array}$} & $\mathrm{G}$ & 8665.86 & 0.018 & 100 & -5.757 \\
\hline July 2 & \multirow[t]{2}{*}{$\mathrm{Pa} 8544$} & G & 8547.34 & 0.051 & 218 & -11.27 & & G & 8667.63 & 0.025 & 235 & -8.323 \\
\hline July 18 & & $\mathrm{G}$ & 8546.9 & 0.069 & 199 & -12.06 & & $\mathrm{G}$ & 8666.86 & 0.033 & 178 & -7.597 \\
\hline June 19 & \multirow{3}{*}{$\mathrm{Pa} 8753$} & $G$ & 8753.51 & 0.004 & 167 & -5.189 & \multirow{3}{*}{$\mathrm{Pa} 8753$} & & $\ldots$ & $\ldots$ & $\ldots$ & $\ldots$ \\
\hline July 2 & & G & 8754.87 & 0.004 & 172 & -2.935 & & G & 8867.5 & 0.006 & 252 & -24.71 \\
\hline July 18 & & G & 8755.33 & 0.007 & 231 & -4.922 & & G & 8868.03 & 0.001 & 141 & -2.992 \\
\hline
\end{tabular}

Table Comments: Measurement errors are $\pm 10 \% .{ }^{a}$ Lorentzian (L), Gaussian (G), complex (c) ${ }^{b}$ Wavelengths are in observer frame.

Figure 3 shows the $0.3-10 \mathrm{keV}$ spectra for all eleven observations, binned for display purposes only, with the best-fit models superimposed. The unabsorbed luminosities are essentially constant (within a factor of $\sim 3$ ) across all observations. The average unabsorbed $0.3-10 \mathrm{keV}$ luminosity is $(3.5 \pm 1.0) \times 10^{39} \mathrm{erg} \mathrm{s}^{-1}$, similar to what was measured by Carpano et al. (2018) and roughly an order of magnitude higher than the classical Eddinginton limit for a neutron star. We can use the luminosity relation $L_{X}=G M_{\mathrm{NS}} \dot{m} R_{\mathrm{NS}}^{-1}$ to estimate the average mass accretion rate onto the ULX-1 neutron star. Assuming canonical neutron star values of $M_{\mathrm{NS}}=1.4 M_{\odot}$ and $R_{\mathrm{NS}}=10 \mathrm{~km}$, we estimate a mass accretion rate $\dot{m} \sim 3 \times 10^{-7} M_{\odot} \mathrm{yr}^{-1}$.

The partial covering fractions vary significantly between observations, from $\sim 78 \%$ on 2016 April 04 to consistent with $\sim 0 \%$ a year later. We define the spectral hardness ratio $H R=(H-S) /(H+S)$, where $H$ is the unabsorbed flux in the $1-10 \mathrm{keV}$ band and $S$ is the unabsorbed flux in the $0.3-1 \mathrm{keV}$ band. There is evidence for changes in the unabsorbed spectral hardness with partial covering fraction (see Figure 4) - the observation with the highest covering fraction exhibits the lowest value of $H R$ (e.g., the unabsorbed hard flux was comparable to the unabsorbed soft flux), while observations with smaller
TABLE 3

Best-Fit 0.3-10 KeV Spectral Model

\begin{tabular}{ccc}
\hline \hline $\begin{array}{c}\text { Component } \\
(1)\end{array}$ & $\begin{array}{c}\text { Parameter } \\
(2)\end{array}$ & $\begin{array}{c}\text { Best-Fit Value } \\
(3)\end{array}$ \\
\hline tbabs & $N_{\mathrm{H}}\left(10^{22} \mathrm{~cm}^{-2}\right)$ & $0.16 \pm 0.03$ \\
pcfabs & $N_{\mathrm{H}}\left(10^{22} \mathrm{~cm}^{-2}\right)$ & $>32.2$ \\
diskbb & $k T_{\text {in }}(\mathrm{keV})$ & $0.40_{-0.06}^{+0.08}$ \\
nthcomp & $\Gamma$ & $1.54_{-0.09}^{+0.11}$ \\
nthcomp & $k T_{e}(\mathrm{keV})$ & $1.76_{-0.41}^{+2.53}$ \\
\hline & $0.3-10 \mathrm{keV}\left(\mathrm{erg} \mathrm{s}^{-1}\right)$ & $(3.5 \pm 1.0) \times 10^{39}$ \\
Luminosity & $0.3-1 \mathrm{keV}\left(\mathrm{erg} \mathrm{s}^{-1}\right)$ & $(7.5 \pm 3.0) \times 10^{38}$ \\
& $1-10 \mathrm{keV}\left(\mathrm{erg} \mathrm{s}^{-1}\right)$ & $(2.8 \pm 1.0) \times 10^{39}$ \\
\hline Photon Flux & $54-200 \mathrm{eV}\left(\mathrm{ph} \mathrm{s}^{-1}\right)$ & $(7.2 \pm 1.3) \times 10^{49}$ \\
\hline & degrees of freedom & 1284 \\
Fit statistics & $C$ & 1053.8 \\
& $\chi_{r}^{2}$ & 1.00 \\
\hline \hline
\end{tabular}

covering fractions exhibit significantly harder values of $H R$.

\subsection{Timing}

X-ray variability provides additional clues to the geometry of supercritical accretion onto the neutron star. Variability can arise from clumps or inhomo- 
TABLE 4

Swift/Xrt Observation Log, Covering Fractions, and Variability

\begin{tabular}{|c|c|c|c|c|c|c|c|c|c|c|c|}
\hline \multirow{2}{*}{$\begin{array}{l}\text { Obs. ID } \\
(1)\end{array}$} & \multirow{2}{*}{$\begin{array}{c}\text { Date } \\
(2)\end{array}$} & \multirow{2}{*}{$\begin{array}{c}\text { Exp. } \\
\text { Time (s) } \\
(3)\end{array}$} & \multicolumn{2}{|c|}{ Position (J2000) } & \multirow{2}{*}{$\begin{array}{c}\text { Net Counts } \\
(0.3-10 \mathrm{keV}) \\
(6)\end{array}$} & \multirow{2}{*}{$\begin{array}{c}\text { covering } \\
\text { fraction }(\%) \\
(7)\end{array}$} & \multirow{2}{*}{$\begin{array}{c}F_{\text {var }} \\
(\%) \\
(8)\end{array}$} & \multicolumn{3}{|c|}{ Luminosity $\left(10^{39} \mathrm{erg} \mathrm{s}^{-1}\right)$} & \multirow{2}{*}{$\begin{array}{l}H R \\
(12)\end{array}$} \\
\hline & & & $\begin{array}{l}\text { R.A. } \\
(4)\end{array}$ & $\begin{array}{c}\text { Decl. } \\
(5)\end{array}$ & & & & $\begin{array}{c}0.3-10 \mathrm{keV} \\
(9)\end{array}$ & $\begin{array}{c}0.3-1 \mathrm{keV} \\
(10)\end{array}$ & $\begin{array}{c}1-10 \mathrm{keV} \\
(11)\end{array}$ & \\
\hline 00049834002 & $2016-04-14$ & 599 & $00: 55: 04.9$ & $-37: 41: 40.2$ & $43 \pm 9$ & $57_{-23}^{+17}$ & $33 \pm 11$ & $2.9_{-2.4}^{+16.2}$ & $0.6_{-0.3}^{+0.4}$ & $2.2_{-0.8}^{+1.1}$ & $0.55 \pm 0.22$ \\
\hline 00049834003 & 2016-04-20 & 489 & 00:55:04.7 & $-37: 41: 40.3$ & $156 \pm 5$ & $78_{-19}^{+12}$ & $31 \pm 8$ & $2.6_{-2.2}^{+14.4}$ & $1.2_{-0.6}^{+0.9}$ & $1.4_{-0.8}^{+1.9}$ & $0.08 \pm 0.04$ \\
\hline 00049834005 & 2016-04-24 & 2922 & 00:55:04.9 & $-37: 41: 46.1$ & $194 \pm 19$ & $59_{-9}^{+8}$ & $33 \pm 11$ & $3.6 \pm 0.5$ & $0.7 \pm 0.2$ & $2.9 \pm 0.5$ & $0.61 \pm 0.10$ \\
\hline 00049834006 & 2017-04-13 & 1128 & 00:55:04.9 & $-37: 41: 41.0$ & $119 \pm$ & $22_{-20}^{17}$ & $32 \pm 11$ & $4.1 \pm 0.7$ & $1.1 \pm 0.3$ & $3.0_{-0.6}^{+0.8}$ & $0.46 \pm 0.08$ \\
\hline 00049834007 & 2017-04-14 & 2545 & $00: 55: 04.8$ & $-37: 41: 46.7$ & $338 \pm 25$ & $16_{-14}^{+12}$ & $29 \pm 12$ & $3.0_{-0.3}^{+0.4}$ & $0.5 \pm 0.2$ & $2.5_{-0.3}^{+0.6}$ & $0.67 \pm 0.14$ \\
\hline 00049834008 & 2017-04-16 & 1853 & 00:55:04.5 & $-37: 41: 48.6$ & $301 \pm 23$ & $<20$ & $34 \pm 9$ & $4.0_{-0.4}^{+0.5}$ & $0.25 \pm 0.03$ & $4.0_{-0.3}^{0^{.5}}$ & $0.93 \pm 0.16$ \\
\hline 00049834009 & 2017-04-21 & 522 & $00: 55: 04.8$ & $-37: 41: 48.6$ & $47 \pm 9$ & $<49$ & $31 \pm 10$ & $2.1_{-0.6}^{+0.4}$ & $0.6_{-0.2}^{+0.3}$ & $1.5_{-0.5}^{+0.3}$ & $0.43 \pm 0.10$ \\
\hline 00049834010 & $2017-04-22$ & 5175 & $00: 55: 04.5$ & $-37: 41: 46.6$ & $617 \pm 33$ & $20 \pm 9$ & $31 \pm 10$ & $6.0_{-3.2}^{+7.0}$ & $1.1_{-0.6}^{+1.5}$ & $4.9_{-2.6}^{+5.6}$ & $0.63 \pm 0.35$ \\
\hline 00049834012 & 2017-07-02 & 1296 & $00: 55: 04.7$ & $-37: 41: 41.2$ & $116 \pm 14$ & $42_{-17}^{+14}$ & $31 \pm 12$ & & $\begin{array}{l}0.7_{-0.2}^{+0.0} \\
0.3\end{array}$ & $2.6_{-0.6}^{+0.0}$ & $0.58 \pm 0.11$ \\
\hline 00049834013 & 2017-07-06 & 1953 & 00:55:04.4 & $-37: 41: 47.0$ & $187 \pm 18$ & $41_{-14}^{+12}$ & $35 \pm 11$ & $3.2_{-0.5}^{+0.0}$ & $0.5 \pm 0.2$ & $2.7 \pm 0.5$ & $0.69 \pm 0.15$ \\
\hline 00049834014 & $2017-07-12$ & 1833 & $00: 55: 04.4$ & $-37: 41: 44.0$ & $156 \pm 17$ & $46_{-13}^{+11}$ & $32 \pm 9$ & $4.2_{-0.6}^{+0.7}$ & $0.8_{-0.2}^{+0.3}$ & $3.3_{-0.6}^{+0.7}$ & $0.60 \pm 0.09$ \\
\hline
\end{tabular}

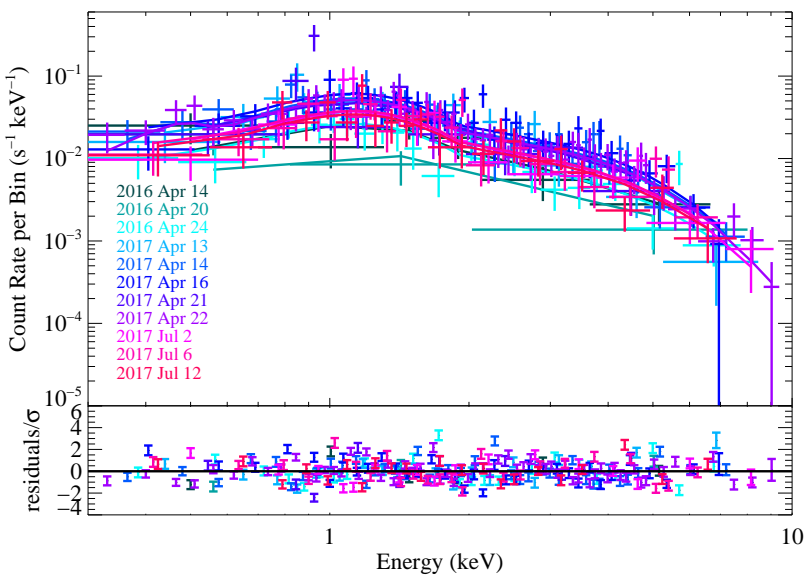

FIG. 3.- The 0.3-10 keV Swift/XRT spectra, with the best-fit model superimposed. Spectra are color-coded by observation date.

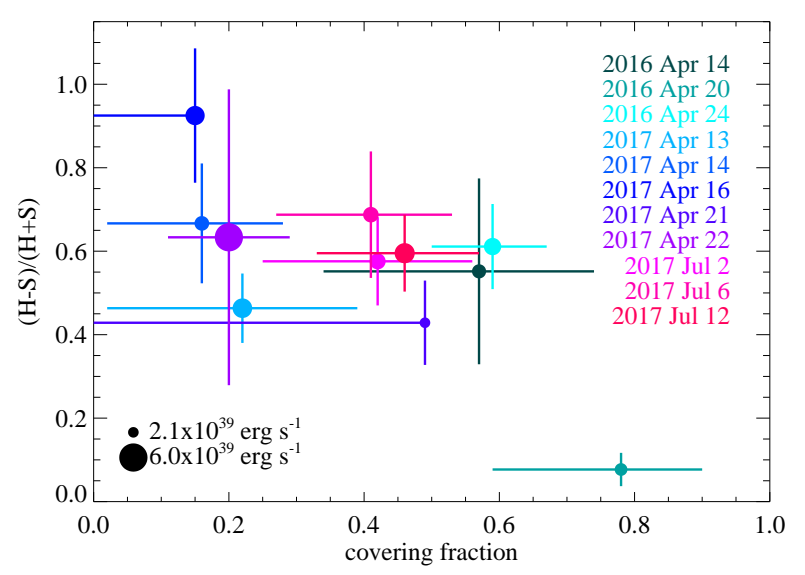

FIG. 4.- The spectral hardness ratio $H R$ as a function of covering fraction. The symbols are scaled to represent the $0.3-10 \mathrm{keV}$ flux, and color-coded according to observation date.

geneities in the winds as they transit the central region from the perspective of the observer. Alternatively, inwardly propagating variations in surface density or mass accretion rate ("flicker noise," e.g., as mass is lost to the wind, Lvubarskii 1997; Ingram \& Done 2012; Middleton et al. 2015) can produce variations in X-ray emission, especially at sub-Eddington ac- cretion rates (Remillard \& McClintock 2006; Heil et al. 2015a, b). Following the approach of Middleton et al. (2015), we compute the fractional root-mean-square variability $\left(F_{\mathrm{var}}\right)$ by Fourier-transforming $\sim 1000 \mathrm{~s}$ segments of the background-subtracted $0.3-10 \mathrm{keV}$ light curves of ULX-1 and averaging the resulting periodograms (see also van der Klis 1989). $\quad F_{\text {var }}$ is then calculated by integrating the power over the frequency range of 3-200 $\mathrm{mHz}$ (where most broad-band variability is observed in ULXs Heil et al. 2009) and taking the square root (Edelson et al. 2002). In all eleven Swift observations, $F_{\text {var }} \sim 30 \%$ (see Table 4 ). Similar variability behavior has been observed in other ULXs with relatively soft spectra (Sutton et al. 2013; Middleton et al. 2015). We used a two-sided K-S test of the photon arrival time in each exposure against the assumption of a constant count rate, but do not find any evidence for significant variability on short time scales. Our light curves (shown in Figure 5) are consistent with a constant count rate, with variations about a constant rate on the order of $\sim 30 \%$.

\section{DISCUSSION}

\subsection{Potential Evidence for Geometric Beaming?}

The observed variation in covering fractions and spectral hardnesses from our X-ray spectral modeling is likely driven by changes in the mass accretion rate onto the ULX-1 neutron star primary. Using the spectraltiming model of Sutton et al. (2013) and Middleton et al. (2015), we can interpret the partial covering component of the spectral model as representing optically thick clumps in the winds of ULX-1 as they obscure the central ionizing source. High covering fractions indicate an increased homogeneity of the winds, and the simultaneous softening of the spectrum may correspond to an increased mass accretion rate. The high luminosities of ULXs have led some to speculate that beaming effects may be important for understanding the geometry of the underlying X-ray binary (King 2009); such beaming would occur if the observer's line of sight to the ULX $(\theta)$ falls within the opening angle over which the wind is launched $\left(\theta_{\mathrm{w}}\right.$;i.e., see Figure 1 in Middleton et al. 2015). At low inclinations, the observer will have a relatively unobstructed view of the hot inner accretion disk and will therefore observe a harder spectrum. Although there is evidence for a relatively hard component in the ULX-1 spectrum $(\Gamma \sim 1.5),>90 \%$ of the unabsorbed flux originates in the soft wind component in all eleven observations. Our X- 

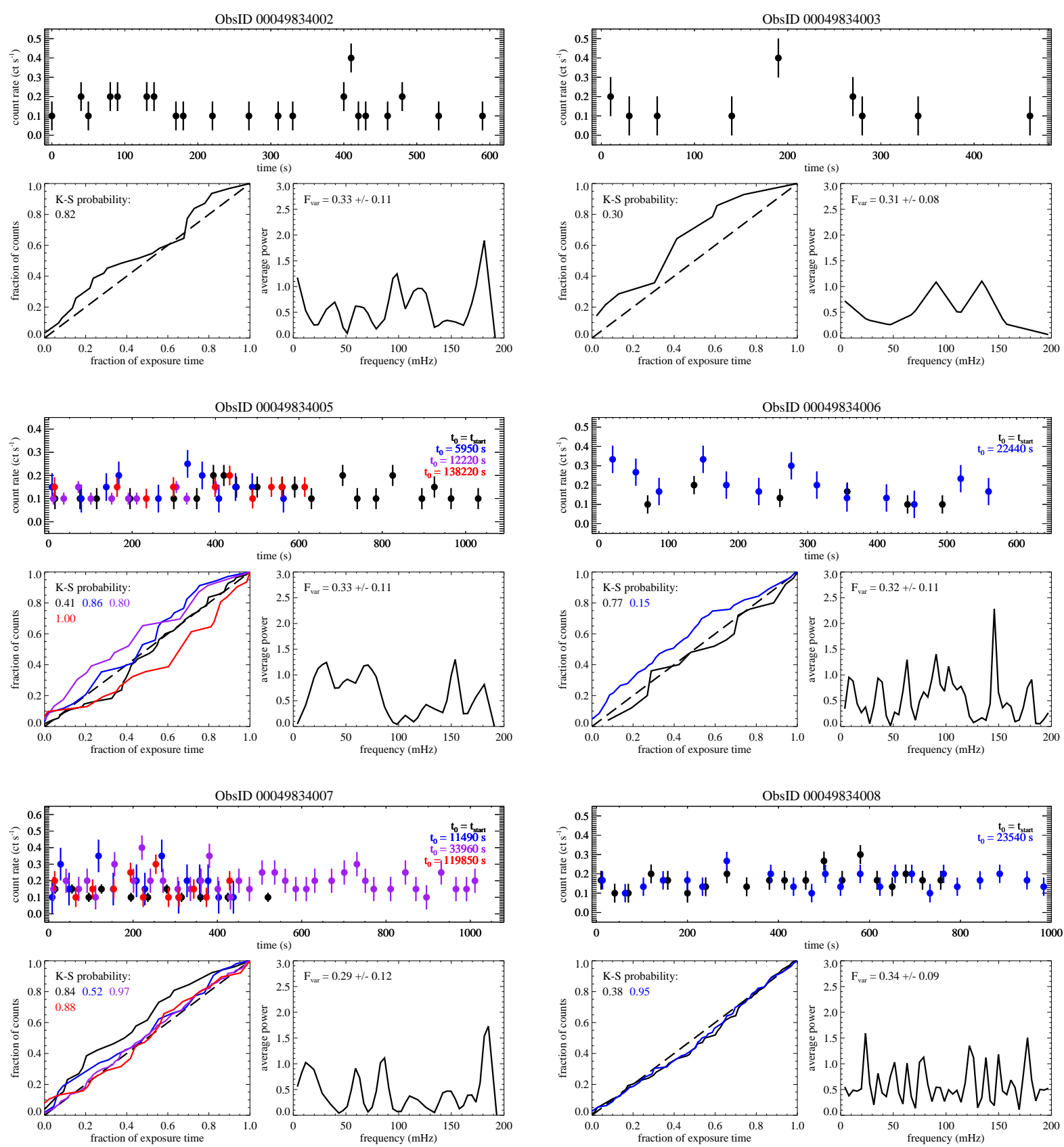

FIG. 5.- Timing analysis plots for the Swift/XRT observations. The top panel shows the 0.3-10 keV light curve. The bottom-left panel shows the photon arrival time (solid line) versus the expectation for a constant count rate (dashed line), used to calculate the K-S probability of non-variability. The bottom right panel shows the $3-200 \mathrm{mHz}$ periodogram, used to calculate $F_{\text {var }}$ (see text for details).

ray spectral-timing suggest that we are observing ULX-1 at small- to moderate- $\theta$.

The importance of beaming was explored for the pulsed neutron star-ULX M82 ULX-2 (King \& Lasota 2016). The relationship between the observed X-ray luminosity and $\dot{m}_{0}$ (the ratio of the thermal timescale mass transfer rate to the Eddington rate) is given by (King 2009; King \& Lasota 2016):

$$
\frac{m_{1}}{L_{40}} \approx \frac{4500}{\dot{m}_{0}^{2}\left(1+\ln \dot{m}_{0}\right)}
$$

where $m_{1}$ is the mass of the neutron star (assumed to be $\left.1.4 M_{\odot}\right)$ and $L_{40}$ is the observed X-ray luminosity in units of $10^{40} \mathrm{erg} \mathrm{s}^{-1}$. Using the average X-ray luminosity from our spectral modeling $\left(L_{40}=0.35\right)$ yields $\dot{m}_{0} \sim 17$. King (2009) give an approximate relationship between $\dot{m}_{0}$ and the beaming factor $b$,

$$
b \approx \frac{73}{\dot{m}_{0}^{2}},
$$

which is valid for $\dot{m}_{0} \gtrsim 8.5$. We therefore estimate a modest beaming factor $b \sim 0.25$ for ULX-1. The "spherization" radius $R_{\text {sph }}$ from which the winds are launched can be estimated from Shakura \& Sunyaev (1973): 

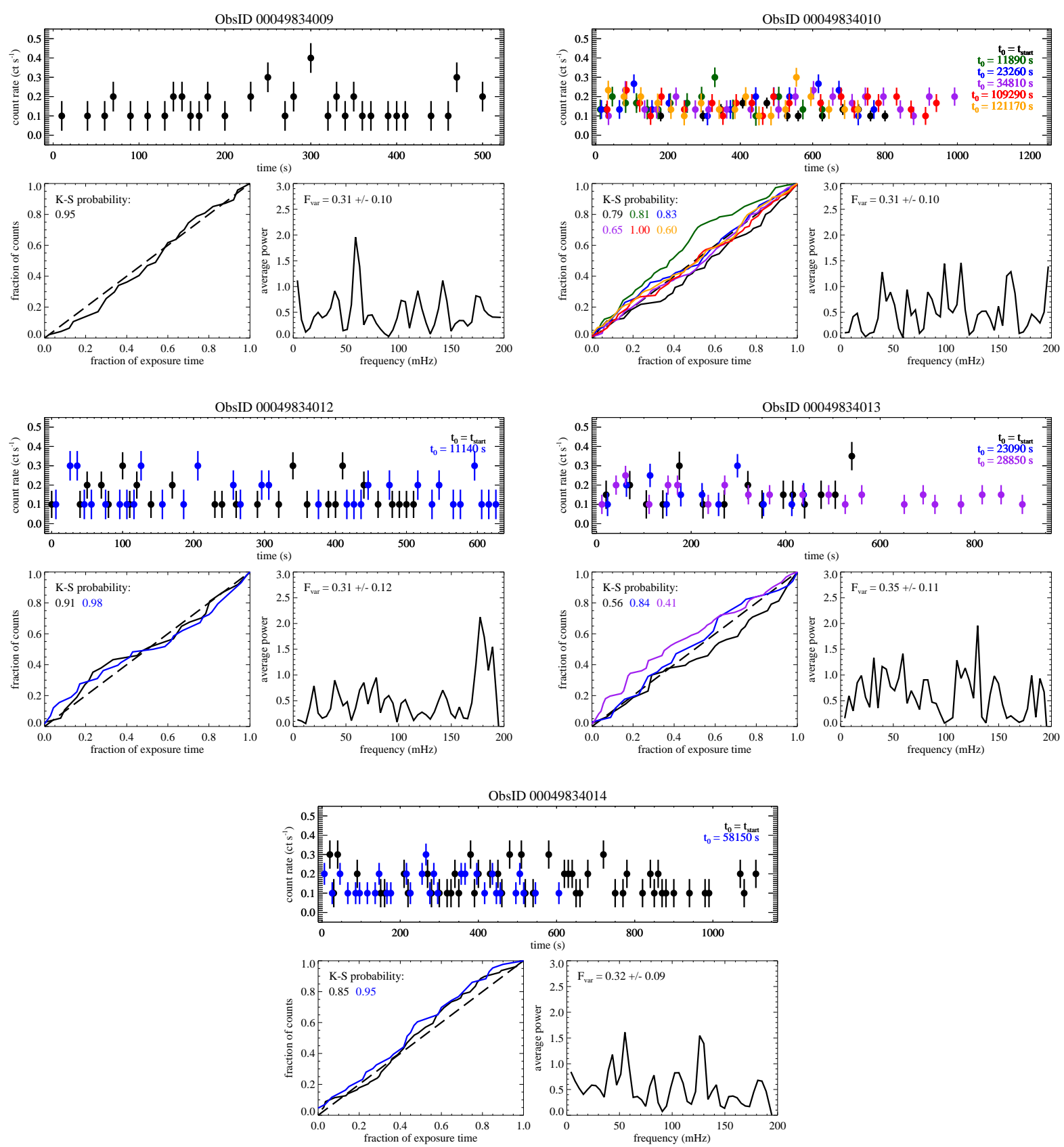

FIG. 5.- Timing analysis plots for the Swift/XRT observations, continued.

$$
R_{\mathrm{sph}} \approx \frac{27}{4} \dot{m}_{0} R_{g}
$$

where $R_{g}$ is the gravitational radius of the neutron star, $R_{g}=G M / c^{2}$. Using this expression, we estimate $R_{\mathrm{sph}} \sim$ $240 \mathrm{~km}$.

Geometric beaming, if present, would further impart evidence on the surrounding material. ULXs are expected to ionize the surrounding ISM (Tarter et al. 1969; Kallman \& McCrav 1982; Pakull \& Angebault 1986) in a manner that is analogous to UV-ionized $\mathrm{H}$ II regions. X-ray ionized nebula, however, will lack a clearly defined boundary (e.g., the equivalent of a Strömgren sphere; Pakull \& Mirioni 2002) and will instead produce an extended, warm region of weakly ionized atoms and collisionally-excited species. The resulting X-ray-ionized nebula will exhibit spectral features from high-ionization species, notably the He II $\lambda 4686$ emission line.

The He II $\lambda 4686$ emission line is particularly sensitive to the photon flux originating from $54-200 \mathrm{eV}$. We measure an average FWHM of $\sim 345 \mathrm{~km} \mathrm{~s}^{-1}$ in the three Gemini spectra where the He II $\lambda 4686$ line is measurable, somewhat higher than the $\sim 270 \mathrm{~km} \mathrm{~s}^{-1} \mathrm{FWHM}$ value found by Villar et al. (2016). Following the approach of Pakull \& Angebault (1986), we can combine our Swift X-ray observations and Gemini optical spectroscopy to determine to what degree beaming is occurring in the ULX-1 system. The 54-200 eV photon flux $(Q)$ can be directly measured from our X-ray spectra. These X-ray 
photons will then excite the He II $\lambda 4686$ emission line in the Gemini spectrum. The absorbed rate of $\mathrm{He}^{+} \mathrm{Ly}_{-}$ man continuum photons, $Q^{\prime}$, in the nebula surrounding ULX-1 can then be calculated by

$$
Q^{\prime}=\frac{L_{4686}}{h v_{4686}} \frac{\alpha_{\mathrm{B}}\left(H e^{+}, T\right)}{\alpha_{4686}^{\mathrm{eff}}(T)},
$$

where $\alpha_{\mathrm{B}}\left(\mathrm{He}^{+}, T\right)$ is the recombination coefficient summed over all levels above the ground state and $\alpha_{4686}^{\text {eff }}(T)$ is the effective recombination coefficient for the emission of He II $\lambda 4686$ photons, which carry an energy $h v_{4686}$. The ratio of these two coefficients is $\sim 5.2$ (Pakull \& Angebault 1986), and depends only weakly on the electron temperature.

The observed He II luminosity from the optical spectrum obtained on 2 July 2017 is $L_{4686} \sim 7-9 \times 10^{35}$ erg $\mathrm{s}^{-1}$, which implies $Q^{\prime} \sim 1.1 \times 10^{48} \mathrm{ph} \mathrm{s}^{-1}$. The Swift X-ray spectrum obtained on the same day yields $Q \sim 6.4 \times 10^{47}$ ph $\mathrm{s}^{-1}$; in general, the X-ray spectra predict an average $Q$ of $(6.8 \pm 2.8) \times 10^{47} \mathrm{ph} \mathrm{s}^{-1}$. The X-ray predicted $Q$ is therefore broadly consistent with the observed $Q^{\prime}$. Any geometric beaming effects, if present in the ULX-1 system at all, are likely small.

\subsection{The Origin of the Optical Emission Lines}

Numerous emission lines are present in the Gemini spectra. Optical images of ULX-1 obtained in 2016 with the Hubble Space Telescope did not show evidence for significant nebulosity - the extinction intrinsic to NGC 300 at the location of the X-ray source was estimated to be $A_{V} \sim 0.4 \mathrm{mag}$ (Binder et al. 2016). Recent work by Lau et al. (2016) and Villar et al. (2016) indicates that dust may be actively reforming in the vicinity of the central binary (within $\sim$ few hundred AU). We therefore expect the emission lines observed in the Gemini spectra originate from the ULX-1 binary or its immediate vicinity, and not from an extended nebula.

The lack of strong [O II] and [S II] lines imply that the material in the immediate vicinity of ULX-1 is not shock-heated (Baldwin et al. 1981; Sutherland \& Dopita 1993; Kewley et al. 2001). The [O III] $\lambda 5007 / \mathrm{H} \beta \sim 0.5$ line ratio and lack of detectable and [N II] $\lambda 6583$ places ULX-1 firmly the photoionization-dominant quadrant of the classic BPT diagram (see, e.g., Evans et al. 1999; Abolmasov et al. 2007, and references therein). This differs from several ULXs that are known to be associated with bright nebulae and bubbles, which typically exhibit spectral features consistent with bright, shock-powered nebulae (Pakull \& Mirioni 2003; Pakull et al.|2006). Ionizing X-ray and UV radiation originating from the 2010 outburst could have affected the surrounding environment only out to a radius of $\sim 8$ light years $(\sim 2.5 \mathrm{pc})$; at the distance of $\mathrm{NGC} \mathrm{300,} \mathrm{this} \mathrm{distance} \mathrm{corresponds}$ to an angular size of $0 .{ }^{\prime \prime} 26$, less than the width of the slit used to obtain the Gemini spectra. The lack of a bright, shock-powered nebula may be due to the young age of the system: ULX-1 is known to be associated with a very young ( $<5 \mathrm{Myr})$ stellar population (Binder et al. 2016). Although this young age is not unique among ULXs (several nearby ULXs also exhibit extremely young ages, consistent with X-ray binary and ULX formation models; Poutanen et al. 2013; Berghea et al. 2013; Wiktorowicz et al. 2017; Linden et al. 2010), most $\mathrm{ULX}_{\mathrm{s}}$ with extended nebulae are $>10 \mathrm{Myr}$ old (see Berghea et al. 2013; Voss et al. 2011; Yang et al. 2011, and references therein) and have been presumably producing ionizing radiation over significantly longer timescales.

$\mathrm{H} \alpha$ is well described by a Lorentzian profile with a full width at half maximum (FWHM) of $\sim 300 \mathrm{~km} \mathrm{~s}^{-1}$ (compared to $\sim 560 \mathrm{~km} \mathrm{~s}^{-1}$ following the initial outburst; Villar et al. 2016). The $\mathrm{H} \alpha$ flux varies by a factor of $\sim 4$ across our observations, ranging from $7.8 \times 10^{-15} \mathrm{erg} \mathrm{s}^{-1}$ $\mathrm{cm}^{-2} \AA^{-1}$ to $3.1 \times 10^{-14} \mathrm{erg} \mathrm{s}^{-1} \mathrm{~cm}^{-2} \AA^{-1}$. In all four observations, the flux ratio $\mathrm{H} \alpha / \mathrm{H} \beta>4$. This is similar to the late-time spectrum obtained by Villar et al. (2016), and is consistent with emission from a persistent wind or mass loss from a blue or yellow supergiant donor star (and not standard case B recombination). A detailed study of the optical spectra will be presented in a forthcoming paper.

\section{CONCLUSIONS}

We have obtained new, near-simultaneous Swift/XRT imaging and Gemini GMOS spectroscopy for the supernova impostor-turned-ULX SN 2010da in NGC 300. The X-ray emission from the object has been persistently high, $\sim 2-6 \times 10^{39} \mathrm{erg} \mathrm{s}^{-1}$, since 2016 , consistent with deep XMM-Newton $+N u S T A R$ observations (Carpano et al. 2018). The X-ray spectra and temporal properties are consistent with the widely accepted "supercritical" model of ULX accretion, in which optically thick winds are launched from a region close to the ionizing central source and reveal a hot, inner accretion disk. The optical spectra suggest that the neutron star X-ray source is photoionizing material in immediate vicinity $(<2.5 \mathrm{pc})$ of the central binary. Our comparison of the soft X-ray emission to the observed He II $\lambda 4686$ emission line luminosity suggests that geometric beaming effects are minimal in the ULX-1 system, making ULX-1 one of only a few bona fide ULXs to be powered by accretion onto a neutron star.

\section{ACKNOWLEDGEMENTS}

This research has made by using Swift/XRT data provided by the UK Swift Science Data Centre at the University of Leicester. The authors would like to thank Loredana Vetere for her assistance obtaining the Swift ToO observations. We thank the Swift team, the PI, the duty scientists and science planners for making the three ToO observations reported here possible. BB was supported by the NSF via award CAREER-1454333 (PI M. Povich).

\section{REFERENCES}

Abolmasov, P., Fabrika, S., Sholukhova, O., \& Afanasiev, V. 2007, Astrophysical Bulletin, 62, 36
Arnaud, K. A. 1996, in Astronomical Society of the Pacific Conference Series, Vol. 101, Astronomical Data Analysis Software and Systems V, ed. G. H. Jacoby \& J. Barnes, 17 
Bachetti, M., et al. 2014, Nature, 514, 202

Baldwin, J. A., Phillips, M. M., \& Terlevich, R. 1981, PASP, 93, 5

Baldwin, J. A., \& Stone, R. P. S. 1984, MNRAS, 206, 241

Berghea, C. T., Dudik, R. P., Tincher, J., \& Winter, L. M. 2013, ApJ, 776, 100

Binder, B., Williams, B. F., Kong, A. K. H., Gaetz, T. J., Plucinsky, P. P., Dalcanton, J. J., \& Weisz, D. R. 2011, ApJ, 739, L51

Binder, B., Williams, B. F., Kong, A. K. H., Gaetz, T. J., Plucinsky, P. P., Skillman, E. D., \& Dolphin, A. 2016, MNRAS, 457, 1636

Brown, P. J. 2010, The Astronomer's Telegram, 2633, 1

Burrows, D. N., et al. 2004, in Proc. SPIE, Vol. 5165, X-Ray and Gamma-Ray Instrumentation for Astronomy XIII, ed. K. A. Flanagan \& O. H. W. Siegmund, 201

Carpano, S., Haberl, F., Maitra, C., \& Vasilopoulos, G. 2018, MNRAS

Chornock, R., \& Berger, E. 2010, The Astronomer's Telegram, 2637, 1

Chornock, R., Czekala, I., \& Berger, E. 2011, The Astronomer's Telegram, 3726, 1

Dalcanton, J. J., et al. 2009, ApJS, 183, 67

Edelson, R., Turner, T. J., Pounds, K., Vaughan, S., Markowitz, A., Marshall, H., Dobbie, P., \& Warwick, R. 2002, ApJ, 568, 610

Elias-Rosa, N., Mauerhan, J. C., \& van Dyk, S. D. 2010a, Central Bureau Electronic Telegrams, 2292, 2

Elias-Rosa, N., Mauerhan, J. C., \& van Dyk, S. D. 2010b, The Astronomer's Telegram, 2636, 1

Evans, I., Koratkar, A., Allen, M., Dopita, M., \& Tsvetanov, Z. 1999, ApJ, 521, 531

Evans, P. A., Beardmore, A. P., Page, K. L., Osborne, J. P. Burrows, D. N., \& Gehrels, N. 2009, in American Institute of Physics Conference Series, Vol. 1133, American Institute of Physics Conference Series, ed. C. Meegan, C. Kouveliotou, \& N. Gehrels, 46

Feng, H., \& Soria, R. 2011, New Astronomy Reviews, 55, 166

Gemini Observatory, \& AURA. 2016, Gemini IRAF: Data reduction software for the Gemini telescopes, Astrophysics Source Code Library

Gimeno, G., et al. 2016, in Proc. SPIE, Vol. 9908, Ground-based and Airborne Instrumentation for Astronomy VI, 99082S

Heil, L. M., Uttley, P., \& Klein-Wolt, M. 2015a, MNRAS, 448, 3348

Heil, L. M., Uttley, P., \& Klein-Wolt, M. 2015b, MNRAS, 448, 3339

Heil, L. M., Vaughan, S., \& Roberts, T. P. 2009, MNRAS, 397, 1061

Hill, J., et al. 2004, in APS Meeting Abstracts

Hook, I. M., Jørgensen, I., Allington-Smith, J. R., Davies, R. L., Metcalfe, N., Murowinski, R. G., \& Crampton, D. 2004, PASP, 116,425

Immler, S., Brown, P., \& Russell, B. R. 2010, The Astronomer's Telegram, 2639, 1

Ingram, A., \& Done, C. 2012, MNRAS, 419, 2369

Kalberla, P. M. W., Burton, W. B., Hartmann, D., Arnal, E. M., Bajaja, E., Morras, R., \& Pöppel, W. G. L. 2005, A\&A, 440, 775
Kallman, T. R., \& McCray, R. 1982, ApJS, 50, 263

Kewley, L. J., Heisler, C. A., Dopita, M. A., \& Lumsden, S. 2001, ApJS, 132, 37

Khan, R., Stanek, K. Z., Kochanek, C. S., Thompson, T. A., \& Prieto, J. L. 2010, The Astronomer's Telegram, 2632, 1

King, A., \& Lasota, J.-P. 2016, MNRAS, 458, L10

King, A. R. 2004, Nuclear Physics B Proceedings Supplements, 132,376

King, A. R. 2009, MNRAS, 393, L41

Kosec, P., Pinto, C., Walton, D. J., Fabian, A. C., Bachetti, M., Fürst, F., \& Grefenstette, B. W. 2018, ArXiv e-prints

Lau, R. M., et al. 2016, ApJ, 830, 142

Linden, T., Kalogera, V., Sepinsky, J. F., Prestwich, A., Zezas, A., \& Gallagher, J. S. 2010, ApJ, 725, 1984

Lyubarskii, Y. E. 1997, MNRAS, 292, 679

Middleton, M. J., Heil, L., Pintore, F., Walton, D. J., \& Roberts, T. P. 2015, MNRAS, 447, 3243

Middleton, M. J., Roberts, T. P., Done, C., \& Jackson, F. E. 2011, MNRAS, 411, 644

Middleton, M. J., Walton, D. J., Roberts, T. P., \& Heil, L. 2014, MNRAS, 438, L51

Monard, L. A. G. 2010, Central Bureau Electronic Telegrams, 2289, 1

Pakull, M. W., \& Angebault, L. P. 1986, Nature, 322, 511

Pakull, M. W., Grisé, F., \& Motch, C. 2006, in IAU Symposium, Vol. 230, Populations of High Energy Sources in Galaxies, ed. E. J. A. Meurs \& G. Fabbiano, 293

Pakull, M. W., \& Mirioni, L. 2002, ArXiv Astrophysics e-prints

Pakull, M. W., \& Mirioni, L. 2003, in Revista Mexicana de Astronomia y Astrofisica Conference Series, Vol. 15, Revista Mexicana de Astronomia y Astrofisica Conference Series, ed. J. Arthur \& W. J. Henney, 197

Poutanen, J., Fabrika, S., Valeev, A. F., Sholukhova, O., \& Greiner, J. 2013, MNRAS, 432, 506

Poutanen, J., Lipunova, G., Fabrika, S., Butkevich, A. G., \& Abolmasov, P. 2007, MNRAS, 377, 1187

Prieto, J. L., Bond, H. E., Kochanek, C. S., Khan, R., Stanek, K. Z., \& Thompson, T. A. 2010, The Astronomer's Telegram, 2660, 1

Remillard, R. A., \& McClintock, J. E. 2006, ARA\&A, 44, 49

Schlafly, E. F., \& Finkbeiner, D. P. 2011, ApJ, 737, 103

Shakura, N. I., \& Sunyaev, R. A. 1973, A\&A, 24, 337

Sutherland, R. S., \& Dopita, M. A. 1993, ApJS, 88, 253

Sutton, A. D., Roberts, T. P., \& Middleton, M. J. 2013, MNRAS, 435,1758

Takeuchi, S., Ohsuga, K., \& Mineshige, S. 2013, PASJ, 65, 88

Takeuchi, S., Ohsuga, K., \& Mineshige, S. 2014, PASJ, 66, 48

Tarter, C. B., Tucker, W. H., \& Salpeter, E. E. 1969, ApJ, 156, 943

van der Klis, M. 1989, ARA\&A, 27, 517

Villar, V. A., et al. 2016, ApJ, 830, 11

Voss, R., Nielsen, M. T. B., Nelemans, G., Fraser, M., \& Smartt, S. J. 2011, MNRAS, 418, L124

Walton, D. J., et al. 2018, ApJ, 857, L3

Wiktorowicz, G., Sobolewska, M., Lasota, J.-P., \& Belczynski, K. 2017, ApJ, 846, 17

Yang, L., Feng, H., \& Kaaret, P. 2011, ApJ, 733, 118 